\title{
Important Paradigms of the Thermoelastic Waves
}

\author{
Ambreen Afsar $\operatorname{Khan}^{a}$, Ayesha Sohail ${ }^{b, c *}$, O A Bég ${ }^{d}$, Rabia Tariq ${ }^{a}$ \\ ${ }^{a}$ Department of Mathematics $\mathcal{G}$ Statistics, International Islamic University, Islamabad 44000, Pakistan \\ ${ }^{b}$ Department of Mathematics, Comsats Institute of Information Technology, Lahore 54000, Pakistan \\ ${ }^{c}$ School of Mathematics and Statistics, The University of Sheffield, S3 7RH, Sheffield, UK \\ ${ }^{d}$ Fluid Mechanics, Spray Research Group, Mechanical and Petroleum Engineering, School of Computing, \\ Science and Engineering, G77, Newton Building, University of Salford, Manchester, M54WT, UK
}

\begin{abstract}
This paper is devoted to the investigation of the propagation of magneto-thermo-elastic waves in a rotating monoclinic system. The system is electrically conducting in the presence of an applied magnetic field. A general dispersion relation is obtained for magneto-thermo-elastic waves. The propagation of wave produced two elastic waves and two thermal waves. It is found that the elastic waves depend on the applied magnetic field and the rotational frequency, where the thermal waves are independent of these effects. The numerical simulations are presented in this article to support the findings.
\end{abstract}

Keywords: Dispersion relation; Thermo-elastic Waves; Rotational frequency; Monoclinic.

\section{Introduction}

There is a strong relation between the dynamical properties of an object and its deformation. The key variables are the change in the temperature and the elastic behaviour, which are closely correlated in the literature to the deformation properties of solids. Investigation of the thermoelastic waves has remained a challenge in industry as well as in the field of bio-informatics and bio-manufacturing, plasma physics and in the study of condensed matter $[1-7,22]$. In short, the theory of elasticity has remained an important area of research since the elementary methods of strength of materials are insufficient at some scales to provide the detailed insight of the stress distribution and other properties in the domain of structural mechanics. To obtain adequate

\footnotetext{
${ }^{*}$ Corresponding author. E-mail address: asohail@ciitlahore.edu.pk
} 
insight of the thermoelastic properties of structures at different scales, it is desired to propose models and to apply quantitative and qualitative methods for analysis.

During the past decade, enormous efforts have been made to address this requirement and the interaction between magnetic fields and strain in a thermoelastic materials is studied due to its many applications [2, 3]. Usually, in these investigations the heat equation under consideration is taken as the uncoupled or the coupled equation [5]. We can summarise the concept of magneto-thermoelasticity as a topic concerned with the relation between magnetic field and electric field in a thermoelastic solid due to its immense applications in the field of geophysics, plasma physics and engineering applications $[6,7,8,9]$. The uncoupled thermo elastic theory has two flaws, first one is that the elastic body has no thermal effect, but it is not experimentally valid. The second one is that any initial change would instantly alter the medium under observation, but physically it is not in accordance with the physical experiments. Thus Biot [10] developed a new thermoelastic theory to remove the first flaw. The heat equation in the Biot theory is still parabolic and thermal wave has an infinite speed. Lord and Shulman [11] proposed the generalized theory of magneto-thermoelasticity with the relaxation times in which the acceleration of heat flux was taken into the account and Fourier law of heat conduction was replaced by generalized version. Green and Naghdi [12-14] re-examined the basic postulates of thermo mechanics, they examined un damped heat wave in an elastic solid and also considered the thermoelastic wave without energy dissipation. Thus they obtained three theories called thermoelasticity of type I, II and III is also known as GN theories I-III. When the three theories are linearized, the heat equation of GN-I is identical to the Fourier law of heat conduction, while GN-II and III showed that the propagation of thermal waves have finite speed.

The combination of three fields such as mechanical (elastic), electromagnetic field and thermal field has attracted considerable attention due to its various applications in science and technology, particular in geophysics, seismology and plasma physics. Knopoff [15] presented the relation between magnetic field and elastic wave motion in electrical conductors. Kaliski and Petykiewicz [16] derived the equation of motion coupled with the field of temperature and magnetic field for an isotropic bodies. Paria [17] investigated the effect of magnetic field on the propagation of thermoelastic waves in an isotropic unbounded medium. After Paria a detailed revision of magneto thermoelastic plane waves were made by Purushothama [18]. He found that when magnetic field is parallel to the direction of wave propagation, the shear wave is purely elastic whereas the compression wave is thermo-elastic in nature. 
The topic of wave propagation in crystalline media shows a very vital role in geophysics and also in ultrasonic and signal processing. The monoclinic crystal is one of the biggest symmetry crystals comprising of almost one third of all the minerals that are present in earth. Singh and Yadav [19] investigated the propagation of plane wave in a monoclinic medium under the effect of magnetic field. They observed that the speed of the wave increases as magnetic field strength increases. Various researchers have investigated the effect of rotation in crystalline media [20,21], with applications in diverse fields of research.

During this research, we have presented the mathematical formulation, which was not available in literature. In this study, we thus present the mathematical formulation to describe the influence of magnetic field on the propagation of thermoelastic wave in a rotating monoclinic medium by using G-N III theory. The monoclinic medium has remained a topic of debate, for many years, to observe the propagation of seismic wave. The magnetothermo-elastic coupled governing equations are established. At the end, the dispersion relation for heat and rotation are obtained separately.

The research during this study is documented in the following order: Section 1 is comprised of the recent literature and the outline of the research methodology. In section 2, we have described the problem by using GN-III theory. The vital feature of GN-III theory is that it sustains dissipation of thermal energy due to the existence of thermal damping term. In section 3 the solution is presented. Section 4 then presents the application of current research on a practical problem.

\section{Formulation of the Problem}

The constitutive relation in a infinite homogeneous elastic solid of monoclinic type having $x_{1}$ plane as the plane of symmetry are given by

$$
\left[\begin{array}{l}
\sigma_{11} \\
\sigma_{22} \\
\sigma_{33} \\
\sigma_{23} \\
\sigma_{13} \\
\sigma_{12}
\end{array}\right]=\left[\begin{array}{cccccc}
C_{11} & C_{12} & C_{13} & C_{14} & 0 & 0 \\
C_{12} & C_{22} & C_{23} & C_{24} & 0 & 0 \\
C_{13} & C_{23} & C_{33} & C_{34} & 0 & 0 \\
C_{14} & C_{24} & C_{34} & C_{44} & 0 & 0 \\
0 & 0 & 0 & 0 & C_{55} & C_{56} \\
0 & 0 & 0 & 0 & C_{56} & C_{66}
\end{array}\right]\left[\begin{array}{c}
\varepsilon_{11} \\
\varepsilon_{22} \\
\varepsilon_{33} \\
2 \varepsilon_{23} \\
2 \varepsilon_{13} \\
2 \varepsilon_{12}
\end{array}\right]
$$

where $2 \varepsilon_{i j}=u_{i, j}+u_{j, i}$ are the components of the displacement vector $\mathbf{v}\left(x_{1}, x_{2}, x_{3}, t\right) . \quad \sigma_{i j}$ are the components of the stress tensor and $C_{i j}(i, j=$ $1,2,3,4,5,6)$ are elastic constants.

Equation governing the propagation of elastic wave in a electrically conduct- 
ing infinite homogeneous monoclinic solid is rotating with an angular velocity $\Omega$ having electromagnetic force $\mathbf{J} \times \mathbf{B}$ (the Lorentz force, $\mathbf{J}$ being the electric current density and $\mathbf{B}$ being the magnetic induction vector)as the body forces are

$$
\sigma_{i j, j}+(\mathbf{J} \times \mathbf{B})_{i}=\rho\left(\ddot{U}_{i}+(\Omega \times(\Omega \times \mathbf{U}))_{i}+2(\Omega \times \dot{\mathbf{U}})_{i}\right),
$$

where $\rho$ is the density of the medium. The Hook's Law for heat conducting material is

$$
\sigma_{i j, j}=C_{i j k l} \varepsilon_{k l}-\gamma \delta_{i j} T \text {. }
$$

The energy equation in the absence of heat source is,

$$
\rho c_{v} \ddot{T}+\gamma T_{0} \ddot{U}_{i, i}=K \dot{T}_{, i i}+K^{*} T_{, i i}
$$

Where $\gamma$ is the thermal modulus, $K$ is the thermal conductivity, $K^{*}$ is a material constant and is the specific heat of the medium.

The well known Maxwell's equations [22] governing the electromagnetic field are,

$$
\begin{gathered}
c u r l \mathbf{H}=\mathbf{J}, \operatorname{div} \mathbf{B}=0, \\
\operatorname{curl} \mathbf{E}=-\frac{\partial \mathbf{B}}{\partial t}, \mathbf{B}=\mu_{e} \mathbf{H}, \mathbf{E}=-\mu_{e}(\dot{\mathbf{U}} \times \mathbf{H}), \\
\mathbf{J}=\sigma\left(\mathbf{E}+\frac{\partial \mathbf{U}}{\partial t} \times \mathbf{B}+(\Omega . \mathbf{B}) \mathbf{U}-(\mathbf{U} . \mathbf{B}) \Omega\right),
\end{gathered}
$$

Here $\mathbf{E}$ is the induced electric field and magnetic field $\mathbf{H}$ induces both primary and induced magnetic field, $\mu_{e}$ and $\sigma$ are the induced permeability and conduction coefficient respectively. Let $\mathbf{H}=\mathbf{H}_{0}+\mathbf{h}$ and $\Omega=\Omega(1,0,0)$ where $\mathbf{H}_{0}=\left(H_{0}, 0,0\right)$. The perturbed magnetic field $\mathbf{h}$ is so small that the product of $\mathbf{U}, \mathbf{h}$, and the derivatives of $\mathbf{h}$ can be ignored when linearizing the field Eqs. (5) to (7) and using the value of $\mathbf{H}$, it gives

$$
\begin{gathered}
\sigma\left(E_{1}\right)=\left(\frac{\partial h_{3}}{\partial x_{2}}-\frac{\partial h_{2}}{\partial x_{3}}\right), \\
\sigma\left(E_{2}+H_{0} \mu_{e} \frac{\partial U_{3}}{\partial t}+\mu_{e} \Omega H_{0} U_{2}\right)=\left(\frac{\partial h_{1}}{\partial x_{3}}-\frac{\partial h_{3}}{\partial x_{1}}\right), \\
\sigma\left(E_{3}-H_{0} \mu_{e} \frac{\partial U_{2}}{\partial t}+\mu_{e} \Omega H_{0} U_{3}\right)=\left(\frac{\partial h_{2}}{\partial x_{1}}-\frac{\partial h_{1}}{\partial x_{2}}\right) .
\end{gathered}
$$


Differentiation of equations 8-10 w.r.t $x_{1}, x_{2}$ and $x_{3}$

The differentiation leads to the following results:

$$
\begin{gathered}
\sigma\left(E_{2,3}+H_{0} \mu_{e} \frac{\partial^{2} U_{3}}{\partial t \partial x_{3}}+\mu_{e} \Omega H_{0} U_{2,3}\right) \\
=\left(\frac{\partial^{2} h_{1}}{\partial x_{3}^{2}}-\frac{\partial^{2} h_{3}}{\partial x_{1} \partial x_{3}}\right), \\
\sigma\left(E_{3,2}-H_{0} \mu_{e} \frac{\partial^{2} U_{2}}{\partial t \partial x_{2}}+\mu_{e} \Omega H_{0} U_{3,2}\right) \\
=\left(\frac{\partial^{2} h_{2}}{\partial x_{1} \partial x_{2}}-\frac{\partial^{2} h_{1}}{\partial x_{2}^{2}}\right), \\
\sigma\left(E_{1,3}\right)=\left(\frac{\partial^{2} h_{3}}{\partial x_{2} \partial x_{3}}-\frac{\partial^{2} h_{2}}{\partial x_{3}^{2}}\right), \\
\sigma\left(E_{3,1}-H_{0} \mu_{e} \frac{\partial^{2} U_{2}}{\partial t \partial x_{1}}+\mu_{e} \Omega H_{0} U_{3,1}\right) \\
=\left(\frac{\partial^{2} h_{2}}{\partial x_{1}^{2}}-\frac{\partial^{2} h_{1}}{\partial x_{2} \partial x_{1}}\right),
\end{gathered}
$$

and

$$
\begin{gathered}
\sigma\left(E_{2,1}+H_{0} \mu_{e} \frac{\partial^{2} U_{3}}{\partial t \partial x_{1}}+\mu_{e} \Omega H_{0} U_{2,1}\right) \\
=\left(\frac{\partial^{2} h_{1}}{\partial x_{3} \partial x_{1}}-\frac{\partial^{2} h_{3}}{\partial x_{1}^{2}}\right), \\
\sigma\left(E_{1,2}\right)=\left(\frac{\partial^{2} h_{3}}{\partial x_{2}^{2}}-\frac{\partial^{2} h_{2}}{\partial x_{3} \partial x_{2}}\right) .
\end{gathered}
$$

where $E_{i, j}=\frac{\partial E_{i}}{\partial x_{j}}$ and $U_{i, j}=\frac{\partial U_{i}}{\partial x_{j}}$ for $i, j=1,2,3$.

\section{Utility of Induced Electric Field}

The formulation for the induced electric field $\mathbf{E}$ can further be written as:

$$
\begin{aligned}
\mathbf{E}_{3,2}-\mathbf{E}_{2,3} & =-\mu_{e} \frac{\partial h_{1}}{\partial t} \\
\mathbf{E}_{1,3}-\mathbf{E}_{3,1} & =-\mu_{e} \frac{\partial h_{2}}{\partial t} \\
\mathbf{E}_{2,1}-\mathbf{E}_{1,2} & =-\mu_{e} \frac{\partial h_{3}}{\partial t}
\end{aligned}
$$


which when interfaced with Eqs (11-16) provides the following set of equations (i.e. Eqs (17-22))

$$
\begin{gathered}
\left(-\frac{\partial h_{1}}{\partial t}-\frac{\partial^{2} U_{2}}{\partial t \partial x_{2}} H_{0}+\Omega H_{0} \frac{\partial U_{3}}{\partial x_{2}}-H_{0} \frac{\partial^{2} U_{3}}{\partial t \partial x_{3}}-\Omega H_{0} \frac{\partial U_{2}}{\partial x_{3}}\right) \\
=\frac{1}{\sigma \mu_{e}}\left(\frac{\partial^{2} h_{2}}{\partial x_{1} \partial x_{2}}-\frac{\partial^{2} h_{1}}{\partial x_{2}^{2}}-\frac{\partial^{2} h_{1}}{\partial x_{3}^{2}}+\frac{\partial^{2} h_{3}}{\partial x_{1} \partial x_{3}}\right), \\
\left(-\frac{\partial h_{2}}{\partial t}-\frac{\partial^{2} U_{2}}{\partial t \partial x_{1}} H_{0}-\Omega H_{0} \frac{\partial U_{3}}{\partial x_{1}}\right)=\frac{1}{\sigma \mu_{e}} \\
\left(\frac{\partial^{2} h_{3}}{\partial x_{2} \partial x_{3}}-\frac{\partial^{2} h_{2}}{\partial x_{3}^{2}}-\frac{\partial^{2} h_{2}}{\partial x_{1}^{2}}+\frac{\partial^{2} h_{1}}{\partial x_{2} \partial x_{1}}\right) \\
\left(-\frac{\partial h_{3}}{\partial t}+\frac{\partial^{2} U_{3}}{\partial t \partial x_{1}} H_{0}+\Omega H_{0} \frac{\partial U_{2}}{\partial x_{1}}\right)=\frac{1}{\sigma \mu_{e}} \\
\left(\frac{\partial^{2} h_{1}}{\partial x_{3} \partial x_{1}}-\frac{\partial^{2} h_{3}}{\partial x_{1}^{2}}-\frac{\partial^{2} h_{3}}{\partial x_{2}^{2}}+\frac{\partial^{2} h_{2}}{\partial x_{3} \partial x_{2}}\right) .
\end{gathered}
$$

\section{Case of Perfect Conduction}

For perfectly conducting material $\sigma \rightarrow \infty$, the Eqs. (17) to (19) becomes

$$
\begin{gathered}
\frac{\partial h_{1}}{\partial t}=H_{0}\left(-\frac{\partial^{2} U_{2}}{\partial t \partial x_{2}}+\Omega \frac{\partial U_{3}}{\partial x_{2}}-\frac{\partial^{2} U_{3}}{\partial t \partial x_{3}}-\Omega \frac{\partial U_{2}}{\partial x_{3}}\right), \\
\frac{\partial h_{2}}{\partial t}=H_{0}\left(\frac{\partial^{2} U_{2}}{\partial t \partial x_{1}}-\Omega \frac{\partial U_{3}}{\partial x_{1}}\right), \\
\frac{\partial h_{3}}{\partial t}=H_{0}\left(\frac{\partial^{2} U_{3}}{\partial t \partial x_{1}}+\Omega \frac{\partial U_{2}}{\partial x_{1}}\right) .
\end{gathered}
$$

\section{Propagation of Elastic Wave, the Component-form and the Alf'ven Wave Velocity}

Then, Eq. (2) in component form can be written as

$$
\begin{gathered}
C_{1}^{2} \frac{\partial^{3} U_{1}}{\partial t \partial x_{1}^{2}}+C_{6}^{2} \frac{\partial^{3} U_{2}}{\partial t \partial x_{2} \partial x_{1}}+C_{5}^{2} \frac{\partial^{3} U_{3}}{\partial t \partial x_{3} \partial x_{1}}+C_{3}^{2} \frac{\partial}{\partial t} \\
\left(\frac{\partial^{2} U_{2}}{\partial x_{3} x_{1}}+\frac{\partial^{2} U_{3}}{\partial x_{2} \partial x_{1}}\right)-\frac{\gamma \partial T}{\rho \partial t \partial x_{1}}+C_{8}^{2} \frac{\partial}{\partial t}\left(\frac{\partial^{2} U_{1}}{\partial x_{2}^{2}}\right.
\end{gathered}
$$




$$
\begin{aligned}
& \left.+\frac{\partial^{2} U_{2}}{\partial x_{1} \partial x_{2}}\right)+C_{7}^{2} \frac{\partial}{\partial t}\left(\frac{\partial^{2} U_{1}}{\partial x_{3} \partial x_{2}}+\frac{\partial^{2} U_{3}}{\partial x_{1} \partial x_{2}}\right)+C_{9}^{2} \frac{\partial}{\partial t} \\
& \left(\frac{\partial^{2} U_{1}}{\partial x_{3}^{2}}+\frac{\partial^{2} U_{3}}{\partial x_{1} \partial x_{3}}\right)+C_{7}^{2} \frac{\partial}{\partial t}\left(\frac{\partial^{2} U_{1}}{\partial x_{2} \partial x_{3}}+\frac{\partial^{2} U_{2}}{\partial x_{1} \partial x_{3}}\right) \\
& =\frac{\partial^{3} U_{1}}{\partial t^{3}} \\
& C_{8}^{2} \frac{\partial}{\partial t}\left(\frac{\partial^{2} U_{1}}{\partial x_{2} \partial x_{1}}+\frac{\partial^{2} U_{2}}{\partial x_{1}^{2}}\right)+C_{7}^{2} \frac{\partial}{\partial t}\left(\frac{\partial^{2} U_{1}}{\partial x_{3} \partial x_{1}}+\frac{\partial^{2} U_{3}}{\partial x_{1}^{2}}\right) \\
& +C_{6}^{2} \frac{\partial^{3} U_{1}}{\partial t \partial x_{1} \partial x_{2}}+C_{10}^{2} \frac{\partial^{3} U_{2}}{\partial t \partial x_{2}^{2}}+C_{11}^{2} \frac{\partial^{3} U_{3}}{\partial t \partial x_{3} \partial x_{2}}+C_{12}^{2} \frac{\partial}{\partial t} \\
& \left(\frac{\partial^{2} U_{2}}{\partial x_{3} \partial x_{2}}+\frac{\partial^{2} U_{3}}{\partial x_{2}^{2}}\right)-\frac{\gamma \partial T}{\rho \partial t \partial x_{2}}+C_{3}^{2} \frac{\partial^{3} U_{1}}{\partial t \partial x_{1} \partial x_{3}}+C_{12}^{2} \\
& \frac{\partial^{3} U_{2}}{\partial t \partial x_{2} \partial x_{3}}+C_{13}^{2} \frac{\partial^{3} U_{3}}{\partial t \partial x_{3}^{2}}+C_{2}^{2} \frac{\partial}{\partial t}\left(\frac{\partial^{2} U_{2}}{\partial x_{3}^{2}}\right. \\
& \left.+\frac{\partial^{2} U_{3}}{\partial x_{2} \partial x_{3}}\right)+C_{A}^{2}\left(\frac{\partial^{3} U_{2}}{\partial t \partial x_{1}^{2}}-\Omega \frac{\partial^{2} U_{3}}{\partial x_{1}^{2}}+\frac{\partial^{3} U_{2}}{\partial t \partial x_{2}^{2}}\right. \\
& \left.-\Omega \frac{\partial^{2} U_{3}}{\partial x_{2}^{2}}+\frac{\partial^{3} U_{3}}{\partial t \partial x_{3} \partial x_{2}}+\Omega \frac{\partial^{2} U_{2}}{\partial x_{3} \partial x_{2}}\right) \\
& =\frac{\partial^{3} U_{2}}{\partial t^{3}}-\Omega^{2} \frac{\partial U_{2}}{\partial t}-2 \Omega \frac{\partial^{2} U_{3}}{\partial t^{2}}, \\
& C_{9}^{2} \frac{\partial}{\partial t}\left(\frac{\partial^{2} U_{1}}{\partial x_{3} \partial x_{1}}+\frac{\partial^{2} U_{3}}{\partial x_{1}^{2}}\right)+C_{7}^{2} \frac{\partial}{\partial t}\left(\frac{\partial^{2} U_{1}}{\partial x_{2} \partial x_{1}}+\frac{\partial^{2} U_{2}}{\partial x_{1}^{2}}\right) \\
& +C_{2}^{2} \frac{\partial}{\partial t}\left(\frac{\partial^{2} \dot{U}_{2}}{\partial x_{2} \partial x_{3}}+\frac{\partial^{2} U_{3}}{\partial x_{2}^{2}}\right)+C_{3}^{2} \frac{\partial^{3} U_{1}}{\partial t \partial x_{1} \partial x_{2}}+C_{12}^{2} \frac{\partial^{3} U_{2}}{\partial t \partial x_{2}^{2}} \\
& +C_{13}^{2} \frac{\partial^{3} U_{3}}{\partial t \partial x_{3} \partial x_{2}}+C_{5}^{2} \frac{\partial^{3} U_{1}}{\partial t \partial x_{1} \partial x_{3}}+C_{11}^{2} \frac{\partial^{3} U_{2}}{\partial t \partial x_{2} \partial x_{3}}+C_{4}^{2} \\
& \frac{\partial^{3} U_{3}}{\partial t \partial x_{3}^{2}}+C_{13}^{2} \frac{\partial}{\partial t}\left(\frac{\partial^{2} \dot{U}_{2}}{\partial x_{3}^{2}}+\frac{\partial^{2} \dot{U}_{3}}{\partial x_{2} \partial x_{3}}\right)-\frac{\gamma \partial T}{\rho \partial t \partial x_{3}}-C_{A}^{2} \\
& \left(-\frac{\partial^{3} U_{2}}{\partial t \partial x_{2} \partial x_{3}}+\Omega \frac{\partial^{2} U_{3}}{\partial x_{2} \partial x_{3}}-\frac{\partial^{3} U_{3}}{\partial t \partial x_{3}^{2}}-\Omega \frac{\partial^{2} U_{2}}{\partial x_{3}^{2}}-\frac{\partial^{3} U_{3}}{\partial t \partial x_{1}^{2}}\right. \\
& \left.-\Omega \frac{\partial^{2} U_{2}}{\partial x_{1}^{2}}\right)=\frac{\partial^{3} U_{3}}{\partial t^{3}}-\Omega^{2} \frac{\partial U_{3}}{\partial t}+2 \Omega \frac{\partial^{2} U_{2}}{\partial t^{2}} .
\end{aligned}
$$

where 


$$
\begin{gathered}
C_{1}^{2}=\frac{C_{11}}{\rho}, C_{2}^{2}=\frac{C_{44}}{\rho}, C_{3}^{2}=\frac{C_{14}}{\rho}, C_{4}^{2}=\frac{C_{33}}{\rho}, \\
C_{5}^{2}=\frac{C_{13}}{\rho}, C_{6}^{2}=\frac{C_{12}}{\rho}, C_{7}^{2}=\frac{C_{56}}{\rho}, C_{8}^{2}=\frac{C_{66}}{\rho}, \\
C_{9}^{2}=\frac{C_{55}}{\rho}, C_{10}^{2}=\frac{C_{22}}{\rho}, C_{11}^{2}=\frac{C_{23}}{\rho}, C_{12}^{2}=\frac{C_{24}}{\rho}, \\
C_{13}^{2}=\frac{C_{34}}{\rho} \text { and } C_{A}^{2}=\frac{\mu_{e} H_{0}^{2}}{\rho},
\end{gathered}
$$

where $C_{A}^{2}$ is the Alf'ven wave velocity (for details, the reader is referred to [23]). Eqs. (23) to (25) can also be written as

$$
\begin{gathered}
\frac{\partial^{3} U_{1}}{\partial t \partial x_{1}^{2}}+\frac{\partial^{3} U_{2}}{\partial t \partial x_{2} \partial x_{1}}\left(V_{5}+V_{7}\right)+\frac{\partial^{3} U_{3}}{\partial t \partial x_{3} \partial x_{1}}\left(V_{1}\right. \\
\left.+V_{8}\right)+\frac{\partial^{3} U_{2}}{\partial t \partial x_{3} \partial x_{1}}\left(V_{4}+V_{6}\right)+\frac{\partial^{3} U_{3}}{\partial t \partial x_{2} \partial x_{1}}\left(V_{4}+V_{6}\right) \\
+\frac{\partial^{3} U_{1}}{\partial t \partial x_{2}^{2}} V_{7}+\frac{\partial^{3} U_{1}}{\partial t \partial x_{3} \partial x_{2}}\left(2 V_{6}\right)+\frac{\partial^{3} U_{1}}{\partial t \partial x_{3}^{2}} V_{8} \\
-\frac{\gamma \partial^{2} T}{\rho C_{1}^{2} \partial t \partial x_{1}}=\frac{1}{C_{1}^{2}} \frac{\partial^{3} U_{1}}{\partial t^{3}} \\
\frac{\partial^{3} U_{1}}{\partial t \partial x_{2} \partial x_{1}}\left(V_{7}+V_{5}\right)+\frac{\partial^{3} U_{2}}{\partial t \partial x_{1}^{2}}\left(V_{7}+R_{H}\right)+\frac{\partial^{3} U_{1}}{\partial t \partial x_{3} \partial x_{1}} \\
\left(V_{6}+V_{4}\right)+\frac{\partial^{3} U_{3}}{\partial t \partial x_{1}^{2}} V_{6}+\frac{\partial^{3} U_{2}}{\partial t \partial x_{2}^{2}}\left(V_{9}+R_{H}\right) \\
+\frac{\partial^{3} U_{3}}{\partial t \partial x_{3} \partial x_{2}}\left(V_{3}+V_{10}+R_{H}\right)+\frac{\partial^{3} U_{2}}{\partial t \partial x_{3} \partial x_{2}}\left(2 V_{11}\right) \\
+\frac{\partial^{3} U_{3}}{\partial t \partial x_{2}^{2}} V_{11}+\frac{\partial^{3} U_{3}}{\partial t \partial x_{3}^{2}} V_{12}+\frac{\partial^{3} U_{2}}{\partial t \partial x_{3}^{2}} V_{3}+R_{H}\left(-\Omega \frac{\partial^{2} U_{3}}{\partial x_{1}^{2}}\right. \\
\left.-\Omega \frac{\partial^{2} U_{3}}{\partial x_{2}^{2}}+\Omega \frac{\partial^{2} U_{2}}{\partial x_{3} \partial x_{2}}\right)-\frac{\gamma \partial^{2} T}{\rho C_{1}^{2} \partial t \partial x_{2}}=\frac{1}{C_{1}^{2}}\left(\frac{\partial^{3} U_{2}}{\partial t^{3}}\right. \\
\left.-\Omega^{2} \frac{\partial U_{2}}{\partial t}-2 \Omega \frac{\partial^{2} U_{3}}{\partial t^{2}}\right), \\
\frac{\partial^{3} U_{1}}{\partial t \partial x_{3} \partial x_{1}}\left(V_{8}+V_{1}\right)+\frac{\partial^{3} U_{3}}{\partial t \partial x_{1}^{2}}\left(V_{8}+R_{H}\right)+\frac{\partial^{3} U_{1}}{\partial t \partial x_{2} \partial x_{1}}
\end{gathered}
$$




$$
\begin{gathered}
\left(V_{6}+V_{4}\right)+\frac{\partial^{3} U_{2}}{\partial t \partial x_{1}^{2}} V_{6}+\frac{\partial^{3} U_{2}}{\partial t \partial x_{2} \partial x_{3}}\left(R_{H}+V_{3}\right. \\
\left.+V_{10}\right)+\frac{\partial^{3} U_{3}}{\partial t \partial x_{2}^{2}} V_{3}+\frac{\partial^{3} U_{2}}{\partial t \partial x_{2}^{2}} V_{11}+\frac{\partial^{3} U_{3}}{\partial t \partial x_{3} \partial x_{2}}\left(2 V_{12}\right) \\
+\frac{\partial^{3} U_{3}}{\partial t \partial x_{3}^{2}}\left(V_{2}+R_{H}\right)+\frac{\partial^{3} U_{2}}{\partial t \partial x_{3}^{2}} V_{12}-R_{H}\left(\Omega \frac{\partial^{2} U_{3}}{\partial x_{2} \partial x_{3}}\right. \\
\left.-\Omega \frac{\partial^{2} U_{2}}{\partial x_{3}^{2}}-\Omega \frac{\partial^{2} U_{2}}{\partial x_{1}^{2}}\right)-\frac{\gamma \partial^{2} T}{\rho C_{1}^{2} \partial t \partial x_{3}}=\frac{1}{C_{1}^{2}}\left(\frac{\partial^{3} U_{3}}{\partial t^{3}}\right. \\
\left.-\Omega^{2} \frac{\partial U_{3}}{\partial t}+2 \Omega \frac{\partial^{2} U_{2}}{\partial t^{2}}\right) .
\end{gathered}
$$

where

$$
\begin{gathered}
V_{1}=\frac{C_{5}^{2}}{C_{1}^{2}}, V_{2}=\frac{C_{4}^{2}}{C_{1}^{2}}, V_{3}=\frac{C_{2}^{2}}{C_{1}^{2}}, V_{4}=\frac{C_{3}^{2}}{C_{1}^{2}}, V_{5}=\frac{C_{6}^{2}}{C_{1}^{2}} \\
V_{6}=\frac{C_{7}^{2}}{C_{1}^{2}}, V_{7}=\frac{C_{8}^{2}}{C_{1}^{2}}, V_{8}=\frac{C_{9}^{2}}{C_{1}^{2}}, V_{9}=\frac{C_{10}^{2}}{C_{1}^{2}}, V_{10}=\frac{C_{11}^{2}}{C_{1}^{2}} \\
V_{11}=\frac{C_{12}^{2}}{C_{1}^{2}}, V_{12}=\frac{C_{13}^{2}}{C_{1}^{2}}, R_{H}=\frac{C_{A}^{2}}{C_{1}^{2}} .
\end{gathered}
$$

where $V_{i}(i=1,2, \ldots, 12)$ are just presenting ratio between the velocity components of the wave in a monoclinic medium, and $R_{H}$ is the magnetic pressure.

The following dimensionless variables and parameters are introduced:

$$
\begin{gathered}
u_{i}=\frac{C_{11}}{\gamma T_{0} l} U_{i}, \xi_{i}=\frac{x_{i}}{l}, \eta=\frac{C_{1} t}{l}, \Omega_{0}=\Omega t_{0} \\
\theta=\frac{T}{T_{0}}, c_{T}^{2}=\frac{K^{*}}{\rho c_{v} C_{1}^{2}}=\frac{c_{3}^{2}}{c_{1}^{2}}, \varepsilon_{T}=\frac{\gamma^{2} T_{0}}{\rho^{2} c_{v} C_{1}^{2}}, \\
k_{0}=\frac{K_{0}}{l C_{1}} \text { and } K_{0}=\frac{K}{\rho c_{V}} .
\end{gathered}
$$

where, $\eta$ is the dimensionless time, $l$ and $t_{0}$ represents the length and time respectively, $K_{0}$ is the thermal diffusivity, $k_{0}$ is the non thermal diffusivity, $c_{T}$ is the thermal velocity and $\varepsilon_{T}$ is the thermo-elastic coupling constant. The details of the constants are provided in [24]. The non-dimensional quantities defined in relation (29), Eqs. (26) to (28) take the form

$$
\frac{\partial^{3} u_{1}}{\partial \eta \partial \xi_{1}^{2}}+\frac{\partial^{3} u_{2}}{\partial \eta \partial \xi_{2} \partial \xi_{1}}\left(V_{5}+V_{7}\right)+\frac{\partial^{3} u_{3}}{\partial \eta \partial \xi_{3} \partial \xi_{1}}\left(V_{1}+V_{8}\right)
$$




$$
\begin{aligned}
& +\frac{\partial^{3} u_{2}}{\partial \eta \partial \xi_{3} \partial \xi_{1}}\left(V_{4}+V_{6}\right)+\frac{\partial^{3} u_{3}}{\partial \eta \partial \xi_{2} \partial \xi_{1}}\left(V_{4}+V_{6}\right) \\
& +\frac{\partial^{3} u_{1}}{\partial \eta \partial \xi_{2}^{2}} V_{7}+\frac{\partial^{3} u_{1}}{\partial \eta \partial \xi_{3} \partial \xi_{2}}\left(2 V_{6}\right)+\frac{\partial^{3} u_{1}}{\partial \eta \partial \xi_{3}^{2}} V_{8} \\
& -\frac{\partial^{2} \theta}{\partial \eta \partial \xi_{1}}=\frac{\partial^{3} u_{1}}{\partial \eta^{3}} \\
& \frac{\partial^{3} u_{1}}{\partial \eta \partial \xi_{2} \partial \xi_{1}}\left(V_{7}+V_{5}\right)+\frac{\partial^{3} u_{2}}{\partial \eta \partial \xi_{1}^{2}}\left(V_{7}+R_{H}\right) \\
& +\frac{\partial^{3} u_{1}}{\partial \eta \partial \xi_{3} \partial \xi_{1}}\left(V_{6}+V_{4}\right)+\frac{\partial^{3} u_{3}}{\partial \eta \partial \xi_{1}^{2}} V_{6}+\frac{\partial^{3} u_{2}}{\partial \eta \partial \xi_{2}^{2}}\left(V_{9}+R_{H}\right) \\
& +\frac{\partial^{3} u_{3}}{\partial \eta \partial \xi_{3} \partial \xi_{2}}\left(V_{3}+V_{10}+R_{H}\right)+\frac{\partial^{3} u_{2}}{\partial \eta \partial \xi_{3} \partial \xi_{2}}\left(2 V_{11}\right) \\
& +\frac{\partial^{3} u_{3}}{\partial \eta \partial \xi_{2}^{2}} V_{11}+\frac{\partial^{3} u_{3}}{\partial \eta \partial \xi_{3}^{2}} V_{12}+\frac{\partial^{3} u_{2}}{\partial \eta \partial \xi_{3}^{2}} V_{3}+R_{H} a\left(-\frac{\partial^{2} u_{3}}{\partial \xi_{1}^{2}}\right. \\
& \left.-\frac{\partial^{2} u_{3}}{\partial \xi_{2}^{2}}+\frac{\partial^{2} u_{2}}{\partial \xi_{3} \partial \xi_{2}}\right)-\frac{\partial^{2} \theta}{\partial \eta \partial \xi_{2}}=\frac{\partial^{3} u_{2}}{\partial \eta^{3}}-a^{2} \frac{\partial u_{2}}{\partial \eta} \\
& -2 a \frac{\partial^{2} u_{3}}{\partial \eta^{2}}, \\
& \frac{\partial^{3} u_{1}}{\partial \eta \partial \xi_{3} \partial \xi_{1}}\left(V_{8}+V_{1}\right)+\frac{\partial^{3} u_{3}}{\partial \eta \partial \xi_{1}^{2}}\left(V_{8}+R_{H}\right) \\
& +\frac{\partial^{3} u_{1}}{\partial \eta \partial \xi_{2} \partial \xi_{1}}\left(V_{6}+V_{4}\right)+\frac{\partial^{3} u_{2}}{\partial \eta \partial \xi_{1}^{2}}\left(V_{6}\right)+\frac{\partial^{3} u_{2}}{\partial \eta \partial \xi_{3} \partial \xi_{2}} \\
& \left(R_{H}+V_{3}+V_{10}\right)+\frac{\partial^{3} u_{3}}{\partial \eta \partial \xi_{2}^{2}}\left(V_{3}\right)+\frac{\partial^{3} u_{2}}{\partial \eta \partial \xi_{2}^{2}}\left(V_{11}\right) \\
& +\frac{\partial^{3} u_{3}}{\partial \eta \partial \xi_{3} \partial \xi_{2}}\left(2 V_{12}\right)+\frac{\partial^{3} u_{3}}{\partial \xi_{3}^{2}}\left(V_{2}+R_{H}\right)+\frac{\partial^{3} u_{2}}{\partial \eta \partial \xi_{3}^{2}} \\
& \left(V_{12}\right)-R_{H} a\left(\frac{\partial^{2} u_{3}}{\partial \xi_{2} \partial \xi_{3}}-\frac{\partial^{2} u_{2}}{\partial \xi_{3}^{2}}-\frac{\partial^{2} u_{2}}{\partial \xi_{1}^{2}}\right) \\
& -\frac{\partial^{2} \theta}{\partial \eta \partial \xi_{3}}=\frac{\partial^{3} u_{3}}{\partial \eta^{3}}-a^{2} \frac{\partial u_{3}}{\partial \eta}+2 a \frac{\partial^{2} u_{2}}{\partial \eta^{2}}, \\
& \frac{\partial^{2} \theta}{\partial \eta^{2}}+\varepsilon_{T}\left(\frac{\partial^{3} u_{1}}{\partial \eta^{2} \partial \xi_{1}}+\frac{\partial^{3} u_{2}}{\partial \eta^{2} \partial \xi_{2}}+\frac{\partial^{3} u_{3}}{\partial \eta^{2} \partial \xi_{3}}\right) \\
& =k_{0}\left(\frac{\partial^{3} \theta}{\partial \eta \partial \xi_{1}^{2}}+\frac{\partial^{2} \theta}{\partial \eta \partial \xi_{2}^{2}}+\frac{\partial^{3} \theta}{\partial \eta \partial \xi_{3}^{2}}\right)
\end{aligned}
$$




$$
+c_{T}^{2}\left(\frac{\partial^{2} \theta}{\partial \xi_{1}^{2}}+\frac{\partial^{2} \theta}{\partial \xi_{2}^{2}}+\frac{\partial^{2} \theta}{\partial \xi_{3}^{2}}\right)
$$

where

$$
a=\frac{\Omega_{0} l}{t_{0} C_{1}} .
$$

\section{Solution of the Problem}

In a manner similar to [25], consider the harmonic plane wave solution of the form,

$$
u_{i}=p_{i} e^{i\left(k \xi_{i} \cdot n_{i}-\omega \eta\right)} \text { and } \theta=\theta_{0} e^{i\left(k \xi_{i} \cdot n_{i}-\omega \eta\right)}
$$

Here $k$ is the wave number, $\omega$ represents the angular frequency of the wave, the direction of wave propagation is represented by the unit vector $n_{i}=\left(n_{1}, n_{2}, n_{3}\right)$ while the direction of particle displacement is denoted by unit vector $p_{i}$. is the wave speed.

Substituting Eq. (34) into Eqs. (30) to (33), we arrive at the system of four homogeneous equations

$$
\begin{gathered}
\left(i \omega k^{2} n_{1}^{2}+\left(i \omega k^{2} n_{2}^{2}\right)\left(V_{7}\right)+\left(2 V_{6}\right)\left(i \omega k^{2} n_{2} n_{3}\right)\right. \\
\left.+\left(i \omega k^{2} n_{3}^{2}\right)\left(V_{8}\right)-i \omega^{3}\right) p_{1} \\
+\left(\left(V_{5}+V_{7}\right)\left(i \omega k^{2} n_{1} n_{2}\right)+\left(V_{4}+V_{6}\right)\left(i \omega k^{2} n_{1} n_{3}\right)\right) p_{2} \\
+\left(\left(V_{1}+V_{8}\right)\left(i \omega k^{2} n_{1} n_{3}\right)+\left(V_{4}+V_{6}\right)\left(i \omega k^{2} n_{1} n_{2}\right)\right) p_{3} \\
-\left(\omega k n_{1}\right) \theta_{0}=0 \\
\left(\left(i \omega k^{2} n_{1} n_{2}\right)\left(V_{5}+V_{7}\right)+\left(i \omega k^{2} n_{1} n_{3}\right)\left(V_{6}+V_{4}\right)\right) p_{1} \\
+\left(\left(i \omega k^{2} n_{1}^{2}\right)\left(V_{7}+R_{H}\right)+\left(V_{9}+R_{H}\right)\left(i \omega k^{2} n_{2}^{2}\right)\right. \\
+\left(i \omega k^{2} n_{2} n_{3}\right)\left(2 V_{11}\right)+\left(i \omega k^{2} n_{3}^{2}\right)\left(V_{3}\right)-R_{H} a\left(k^{2} n_{2} n_{3}\right) \\
\left.-\left(i \omega^{3}\right)-a^{2}(i \omega)\right) p_{2}+\left(( i \omega k ^ { 2 } n _ { 2 } n _ { 3 } ) \left(V_{3}+V_{10}\right.\right. \\
\left.+R_{H}\right)+\left(i \omega k^{2} n_{2}^{2}\right)\left(V_{11}\right)+\left(i \omega k^{2} n_{3}^{2}\right)\left(V_{12}\right)
\end{gathered}
$$




$$
\begin{gathered}
\left.+R_{H}\left(a k^{2} n_{1}^{2}\right)+R_{H} a\left(k^{2} n_{2}^{2}\right)-2 a\left(\omega^{2}\right)\right) p_{3} \\
-\left(\omega k n_{2}\right) \theta_{0}=0 \\
\left(\left(i \omega k^{2} n_{1} n_{3}\right)\left(V_{1}+V_{8}\right)+\left(i \omega k^{2} n_{1} n_{2}\right)\left(V_{6}+V_{4}\right)\right) p_{1} \\
+\left(i \omega k^{2} n_{1}^{2}\right)\left(\left(V_{6}\right)+\left(i \omega k^{2} n_{2} n_{3}\right)\left(R_{H}+V_{3}+V_{10}\right)\right. \\
+\left(i \omega k^{2} n_{2}^{2}\right)\left(V_{11}\right)+\left(i \omega k^{2} n_{3}^{2}\right)\left(V_{12}\right)-R_{H} a\left(k^{2} n_{3}^{2}\right) \\
\left.-R_{H} a\left(k^{2} n_{1}^{2}\right)+2 a\left(\omega^{2}\right)\right) p_{2} \\
+\left(\left(i \omega k^{2} n_{1}^{2}\right)\left(V_{8}+R_{H}\right)+\left(i \omega k^{2} n_{2}^{2}\right)\left(V_{3}\right)+\left(i \omega k^{2} n_{2} n_{3}\right)\right. \\
\left(2 V_{12}\right)+\left(i \omega k^{2} n_{3}^{2}\right)\left(V_{2}+R_{H}\right)+R_{H} a\left(k^{2} n_{2} n_{3}\right) \\
\left.-\left(i \omega^{3}\right)-a^{2}(i \omega)\right) p_{3}-\left(\omega k n_{3}\right) \theta_{0}=0 \\
\left(\varepsilon_{T} n_{1} \omega^{2}\right) p_{1}+\left(\varepsilon_{T} n_{2} \omega^{2}\right) p_{2}+\left(\varepsilon_{T} n_{3} \omega^{2}\right) p_{3} \\
+\left(k_{0} \omega k+i k c_{T}^{2}-\frac{i \omega^{2}}{k}\right) \theta_{0}=0 .
\end{gathered}
$$

The system of equations has non trivial solution if and only if the determination of the factor matrix vanishes. So

$$
\left|\begin{array}{llll}
A_{11} & A_{12} & A_{13} & A_{14} \\
A_{21} & A_{22} & A_{23} & A_{24} \\
A_{31} & A_{32} & A_{33} & A_{34} \\
A_{41} & A_{42} & A_{43} & A_{44}
\end{array}\right|=0
$$

where

$$
\begin{gathered}
A_{11}=i \omega k^{2} n_{1}^{2}+\left(i \omega k^{2} n_{2}^{2}\right)\left(V_{7}\right)+\left(2 V_{6}\right)\left(i \omega k^{2} n_{2} n_{3}\right) \\
+\left(i \omega k^{2} n_{3}^{2}\right)\left(V_{8}\right)-i \omega^{3}, \\
A_{12}=\left(V_{5}+V_{7}\right)\left(i \omega k^{2} n_{1} n_{2}\right)+\left(V_{4}+V_{6}\right)\left(i \omega k^{2} n_{1} n_{3}\right), \\
A_{13}=\left(V_{1}+V_{8}\right)\left(i \omega k^{2} n_{1} n_{3}\right)+\left(V_{4}+V_{6}\right)\left(i \omega k^{2} n_{1} n_{2}\right),
\end{gathered}
$$




$$
\begin{gathered}
A_{14}=-\omega k n_{1}, \quad A_{24}=-\omega k n_{2}, \quad A_{34}=-\omega k n_{3}, \\
A_{21}=\left(i \omega k^{2} n_{1} n_{2}\right)\left(V_{5}+V_{7}\right)+\left(i \omega k^{2} n_{1} n_{3}\right)\left(V_{6}+V_{4}\right), \\
A_{22}=\left(i \omega k^{2} n_{1}^{2}\right)\left(V_{7}+R_{H}\right)+\left(V_{9}+R_{H}\right)\left(i \omega k^{2} n_{2}^{2}\right)+\left(i \omega k^{2} n_{2} n_{3}\right) \\
\left(2 V_{11}\right)+\left(i \omega k^{2} n_{3}^{2}\right)\left(V_{3}\right)-R_{H} a\left(k^{2} n_{2} n_{3}\right)-\left(i \omega^{3}\right)-a^{2}(i \omega), \\
A_{23}=\left(i \omega k^{2} n_{2} n_{3}\right)\left(V_{3}+V_{10}+R_{H}\right)+\left(i \omega k^{2} n_{2}^{2}\right)\left(V_{11}\right)+\left(i \omega k^{2} n_{3}^{2}\right) \\
\left(V_{12}\right)+R_{H}\left(a k^{2} n_{1}^{2}\right)+R_{H} a\left(k^{2} n_{2}^{2}\right)-2 a\left(\omega^{2}\right), \\
A_{31}=\left(i \omega k^{2} n_{1} n_{3}\right)\left(V_{1}+V_{8}\right)+\left(i \omega k^{2} n_{1} n_{2}\right)\left(V_{6}+V_{4}\right), \\
\left.A_{32}=i \omega k^{2} n_{1}^{2}\right)\left(\left(V_{6}\right)+\left(i \omega k^{2} n_{2} n_{3}\right)\left(R_{H}+V_{3}+V_{10}\right)+\left(i \omega k^{2} n_{2}^{2}\right)\right. \\
\left(V_{11}\right)+\left(i \omega k^{2} n_{3}^{2}\right)\left(V_{12}\right)-R_{H} a\left(k^{2} n_{3}^{2}\right)-R_{H} a\left(k^{2} n_{1}^{2}\right)+2 a\left(\omega^{2}\right), \\
A_{33}=\left(i \omega k^{2} n_{1}^{2}\right)\left(V_{8}+R_{H}\right)+\left(i \omega k^{2} n_{2}^{2}\right)\left(V_{3}\right)+\left(i \omega k^{2} n_{2} n_{3}\right)\left(2 V_{12}\right) \\
+\left(i \omega k^{2} n_{3}^{2}\right)\left(V_{2}+R_{H}\right)+R_{H} a\left(k^{2} n_{2} n_{3}\right)-\left(i \omega^{3}\right)-a^{2}(i \omega), \\
A_{41}=\varepsilon_{T} n_{1} \omega^{2}, \quad A_{42}=\varepsilon_{T} n_{2} \omega^{2}, A_{43}=\varepsilon_{T} n_{3} \omega^{2}, \\
A_{44}=k_{0} \omega k+i k c_{T}^{2}-\frac{i \omega^{2}}{k} .
\end{gathered}
$$

For simplicity, we choose the propagating vector along $x_{1}$ axis i.e., $\mathbf{n}=$ $(1,0,0)$, the dispersion relation can be converted to the following form.

$$
\begin{gathered}
{\left[k^{4}\left(a^{2} R_{H}^{2}+V_{6}^{2} \omega^{2}-V_{8} R_{H} \omega^{2}-R_{H}^{2} \omega^{2}-V_{8} V_{7} \omega^{2}-R_{H} V_{7} \omega^{2}\right)\right.} \\
+k^{2}\left(a^{2} V_{8} \omega^{2}-4 a^{2} R_{H} \omega^{2}+2 a^{2} R_{H} \omega^{2}+a^{2} V_{7} \omega^{2}+V_{8} \omega^{4}+V_{7} \omega^{4}\right. \\
\left.\left.\quad+2 R_{H} \omega^{4}\right)+\left(-a^{4} \omega^{2}+4 a^{2} \omega^{4}-2 a^{2} \omega^{4}-\omega^{6}\right)\right] \\
{\left[k^{4}\left(i k_{0} \omega-c_{T}^{2}\right)+k^{2}\left(\omega^{2}\left(\varepsilon+c_{T}^{2}+1\right)-i k_{0} \omega^{3}\right)-\omega^{4}\right]=0 .}
\end{gathered}
$$

The above equation is the dispersion relation for plane wave propagation in a monoclinic medium. It has two factors. $1^{\text {st }}$ factor depends on the magnetic field and frequency of rotation and $2^{\text {nd }}$ factor depends on the thermal velocity and angular velocity of the elastic wave. 


\section{Practical Application}

For the case of monoclinic medium, we have considered the following parametric values:

$$
\begin{aligned}
& \rho=1.74 \times 10^{3} \mathrm{~kg} / \mathrm{m}^{3}, C_{11}=5.974 \times 10^{10} \mathrm{~N} / \mathrm{m}^{2}, C_{66}=0.93 \times 10^{11} \mathrm{~N} / \mathrm{m}^{2}, \\
& C_{55}=0.94 \times 10^{11} \mathrm{~N} / \mathrm{m}^{2}, C_{56}=-0.11 \times 10^{11} \mathrm{~N} / \mathrm{m}^{2} .
\end{aligned}
$$

These values were selected from the results presented by Chattopadhyay et'al. [21].

The magnetic field is given by the following relation as, $B=\mu_{0}\left(1+x_{m}\right) H$, where $\mu_{e}=\mu_{0}\left(1+x_{m}\right)$ is the magnetic permeability, $x_{m}$ is the constant for magnetic susceptibility whose value depends on the nature of the material, $\mu_{0}$ is the free space magnetic permeability. For sulfur $x_{m}$ is $14.9 \times 10^{-6}$ and $\mu_{0}$ is $4 \pi \times 10^{-7} \mathrm{Hm}^{-1}$ [26], therefore, the relative permeability is equal to 1 . From Eq. (39),

$$
\begin{gathered}
{\left[k ^ { 4 } \left(a^{2} R_{H}^{2}+V_{6}^{2} \omega^{2}-V_{8} R_{H} \omega^{2}-R_{H}^{2} \omega^{2}-V_{8} V_{7} \omega^{2}\right.\right.} \\
\left.-R_{H} V_{7} \omega^{2}\right)+k^{2}\left(a^{2} V_{8} \omega^{2}-4 a^{2} R_{H} \omega^{2}+2 a^{2} R_{H} \omega^{2}\right. \\
\left.+a^{2} V_{7} \omega^{2}+V_{8} \omega^{4}+V_{7} w^{4}+2 R_{H} \omega^{4}\right)+\left(-a^{4} \omega^{2}+4 a^{2} \omega^{4}\right. \\
\left.\left.-2 a^{2} \omega^{4}-\omega^{6}\right)\right]=0
\end{gathered}
$$

This is the quadratic equation in $k^{2}$ so having two roots of velocity of waves, the values of these velocities are depending on the strength of magnetic field and frequency of rotation. Figs. 1 and 2 depicts the behaviour of wave velocity relative to frequency of rotation under the variation of $H_{0}$. Fig. 1 shows that the wave velocity show peaks at two different values of a, one between 0.5 to 1 and other between 2 to 2.5. The first peak increase it maximum value for stronger value of magnetic field $H_{0}$, while the second peak shows that the opposite trend. Fig. 2 depicts that the wave velocity is decreases with increasing rotation a.

And,

$$
\left[k^{4}\left(i k_{0} \omega-c_{T}^{2}\right)+k^{2}\left(\omega^{2}\left(\varepsilon+c_{T}^{2}+1\right)-i k_{0} \omega^{3}\right)-\omega^{4}\right]=0
$$

This is the quadratic equation in $k^{2}$ so having two roots, which represents existence of two thermal waves. Fig. 3, 4, 5 and 6 demonstrate the effects of coupling factor $\varepsilon_{T}$ and thermal diffusivity $k_{0}$ on the wave velocity. Figs. 3 and 4 is a graph for one wave and Figs. 5 and 6 is for $2^{\text {nd }}$ wave. It is 


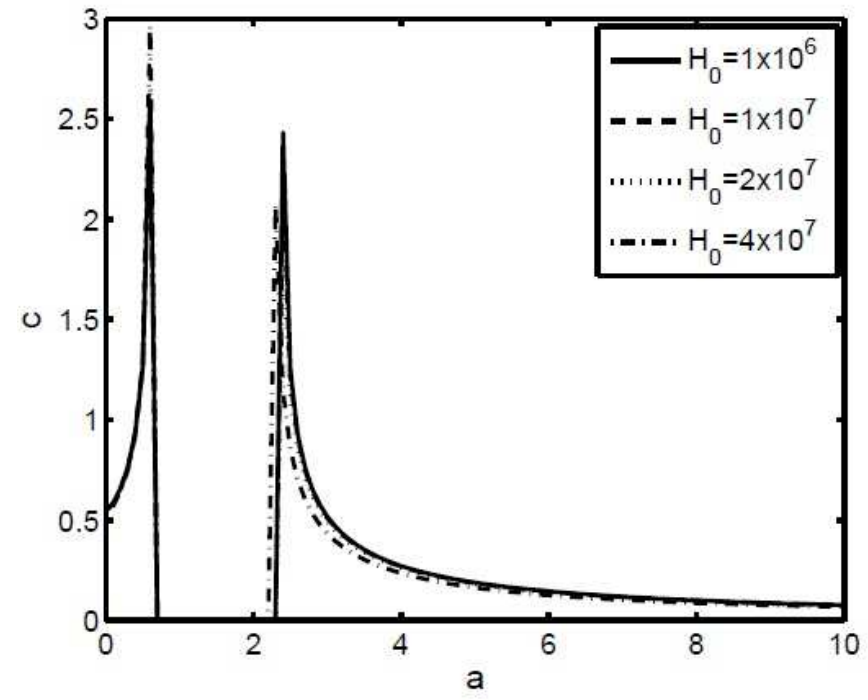

Figure 1: The wave velocity $c(\mathrm{~m} / \mathrm{s})$ versus rotation $a(\mathrm{~Hz})$ for different values of $H_{0}$ when $\omega=1$.

perceived from these Figs. that, the wave velocity attain its maximum value before the thermal velocity attain its value 2 . In both cases wave velocity decreases on increasing $\varepsilon_{T}$ and $k_{0}$.

\section{Conclusion}

This paper presented the propagation of an elastic wave in a rotating monoclinic medium in the presence of magnetic field. Thus our results provides an evidence that in the case of energy dissipation diffusivity is ignored. It is examined through graphs that energy dissipation changes the nature of the curve. The elastic wave velocity becomes independent of diffusivity for higher values of thermal velocity. Furthermore, the elastic wave velocity declines by increasing the magnetic field and coupling constant.

Dispersion relations for thermoelastic wave has been derived. The main findings are summarized as:

1) For higher values of thermal velocity $c_{T}$, the elastic wave velocity $c$ becomes independent of diffusivity.

2 ) Increase in the couple factor $\varepsilon_{T}$, decreases the wave velocity $c$.

3) The wave velocity decreases with increasing magnetic field. 


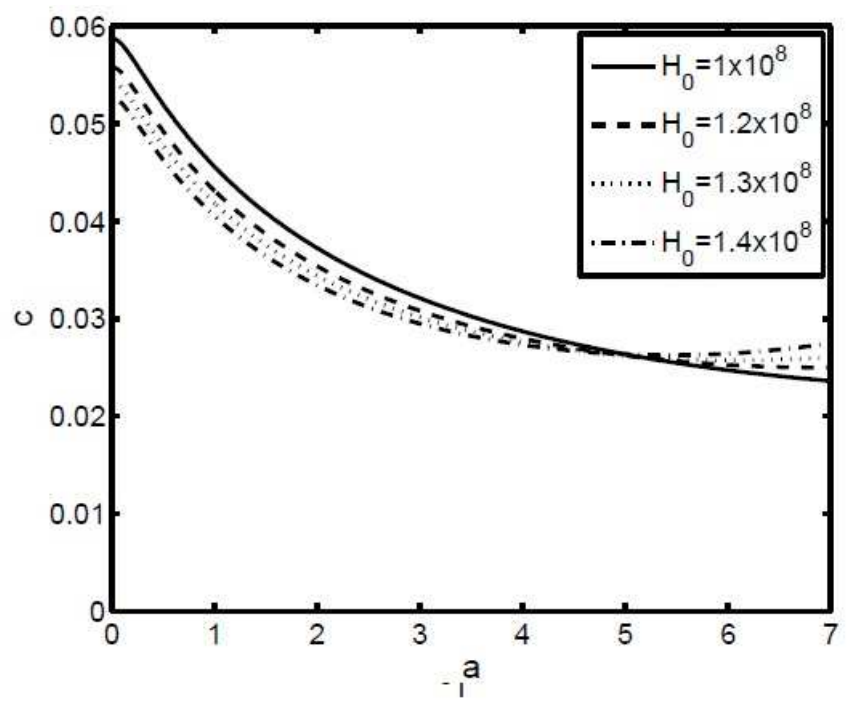

Figure 2: The wave velocity $c(\mathrm{~m} / \mathrm{s})$ versus rotation $a(\mathrm{~Hz})$ for different values of $H_{0}$ when $\omega=1$.

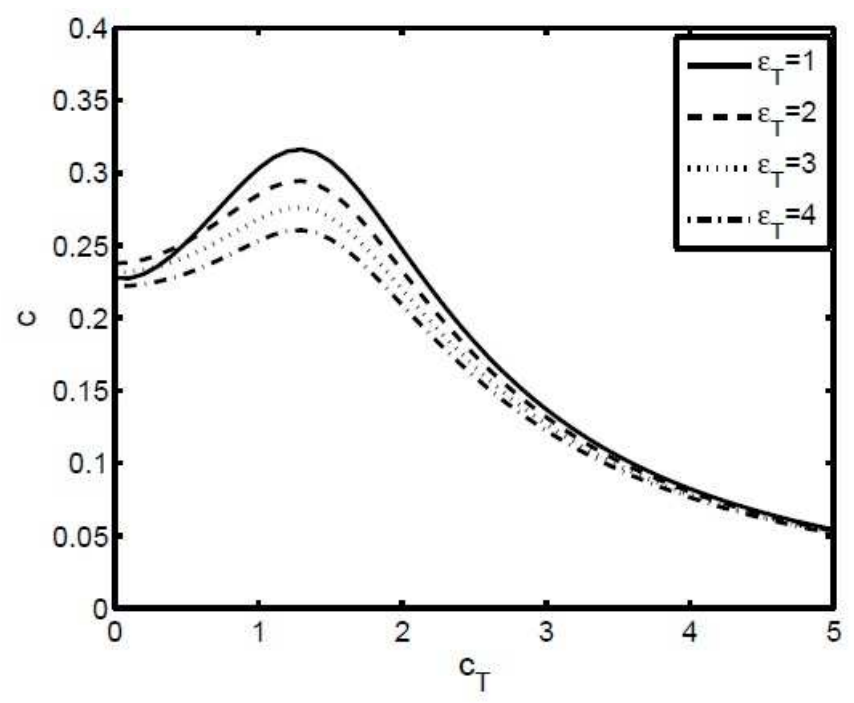

Figure 3: The wave velocity $c(\mathrm{~m} / \mathrm{s})$ versus thermal velocity $c_{T}(\mathrm{~m} / \mathrm{s})$ for different values of $\varepsilon_{T}$ when $\omega=1, k_{0}=2$. 


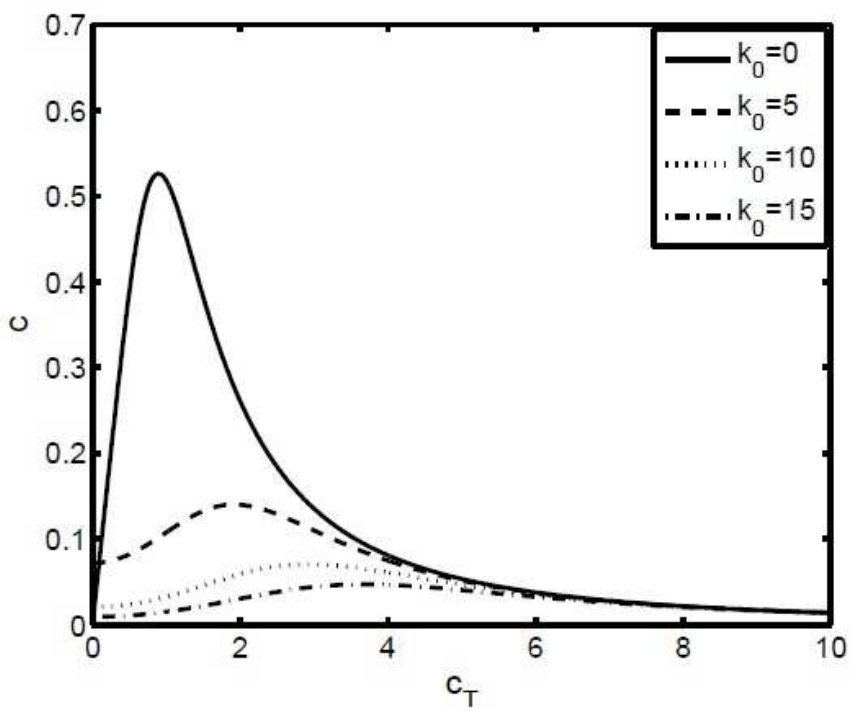

Figure 4: The wave velocity $c$ versus thermal velocity $c_{T}$ for different values of $k_{0}$ when $\omega=1, \varepsilon_{T}=2$.

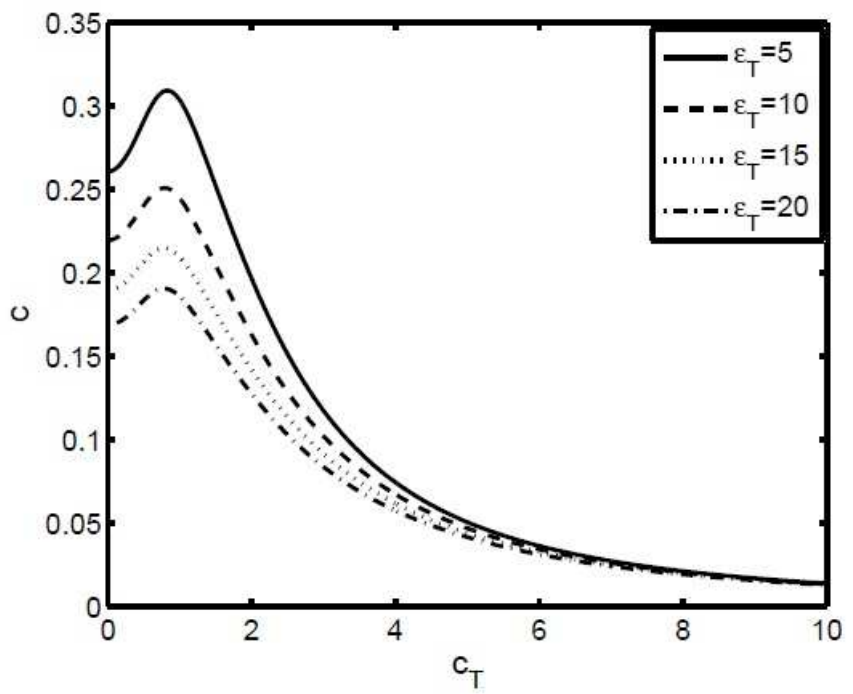

Figure 5: The wave velocity $c$ versus thermal velocity $c_{T}$ for different values of $\varepsilon_{T}$ when $\omega=1, k_{0}=2$. 


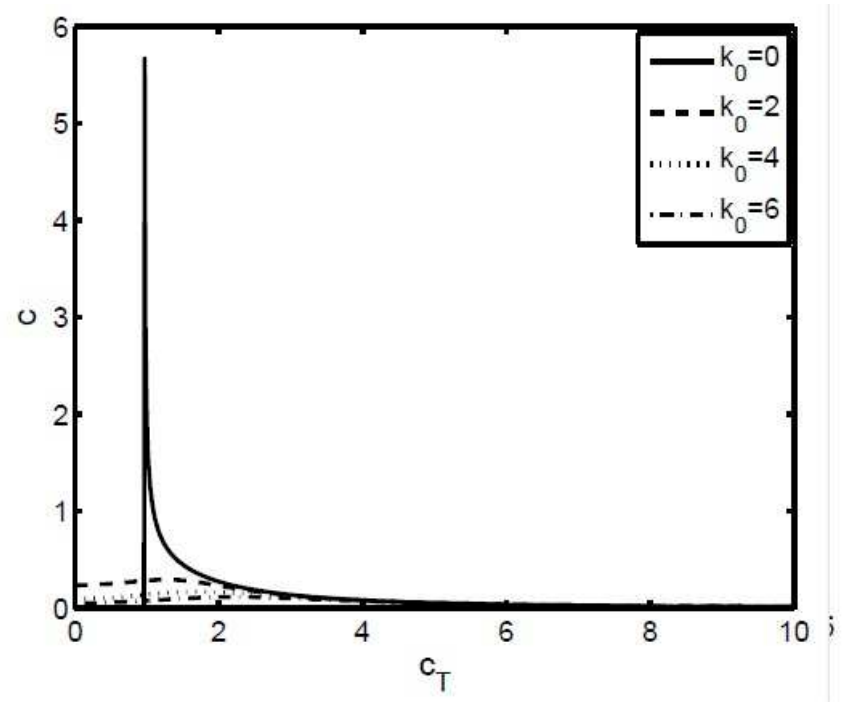

Figure 6: The wave velocity $c$ versus thermal velocity $c_{T}$ for different values of $k_{0}$ when $\omega=1, \varepsilon_{T}=2$.

\section{References}

[1] Ezzat, M.A., 2008. State space approach to solids and fluids. Canadian Journal of Physics, 86(11), pp.1241-1250.

[2] Ezzat, M.A. and El-Bary, A.A., 2017. Application of fractional order theory of magneto-thermoelasticity to an infinite perfect conducting body with a cylindrical cavity. Microsystem Technologies, 23(7), pp.24472458 .

[3] Arshad, S., Siddiqui, A.M., Sohail, A., Maqbool, K. and Li, Z., 2017. Comparison of optimal homotopy analysis method and fractional homotopy analysis transform method for the dynamical analysis of fractional order optical solitons. Advances in Mechanical Engineering, 9(3), p.1687814017692946.

[4] Abbas, I.A. and Abo-Dahab, S.M., 2014. On the numerical solution of thermal shock problem for generalized magneto-thermoelasticity for an infinitely long annular cylinder with variable thermal conductivity. Journal of Computational and Theoretical Nanoscience, 11(3), pp.607618. 
[5] Wajid, H.A. and Sohail, A., 2016. Compact Modified Implicit Finite Element Schemes for Wave Propagation Problems with Superior Dispersive Properties. Arabian Journal for Science and Engineering, 41(11), pp.4613-4624.

[6] Sohail, A., Rees, J.M. and Zimmerman, W.B., 2011. Analysis of capillary-gravity waves using the discrete periodic inverse scattering transform. Colloids and Surfaces A: Physicochemical and Engineering Aspects, 391(1-3), pp.42-50.

[7] Cherifi, R.O., Ivanovskaya, V., Phillips, L.C., Zobelli, A., Infante, I.C., Jacquet, E., Garcia, V., Fusil, S., Briddon, P.R., Guiblin, N. and Mougin, A., 2014. Electric-field control of magnetic order above room temperature. Nature materials, 13(4), p.345.

[8] Kittel, C., McEuen, P. and McEuen, P., 1996. Introduction to solid state physics (Vol. 8, pp. 323-324). New York: Wiley.

[9] Han, T., Bai, X., Gao, D., Thong, J.T., Li, B. and Qiu, C.W., 2014. Experimental demonstration of a bilayer thermal cloak. Physical review letters, 112(5), p.054302.

[10] Biot, M.A., 1956. Thermoelasticity and irreversible thermodynamics. Journal of Applied Physics, 27(3), pp.240-253.

[11] Lord, H.W. and Shulman, Y., 1967. A generalized dynamical theory of thermoelasticity. Journal of the Mechanics and Physics of Solids, 15(5), pp.299-309.

[12] Green, A.E. and Naghdi, P.M., 1991, February. A re-examination of the basic postulates of thermomechanics. In Proc. R. Soc. Lond. A (Vol. 432, No. 1885, pp. 171-194). The Royal Society.

[13] Green, A.E. and Naghdi, P.M., 1992. An Unbounded Heat Wave in an Elastic Solid. Journal of Thermal Stresses, 15 pp 253-264.

[14] Green, A.E. and Naghdi, P.M., 1993. Thermoelasticity Without Energy Dissipation, 31, pp189-208.

[15] Knopoff, L., 1955. The interaction between elastic wave motions and a magnetic field in electrical conductors. Journal of Geophysical Research, 60(4), pp.441-456. 
[16] S. Kaliski and J. Petykiewicz, 1959 Equation of motion coupled with the field of temperature in a magnetic field involving mechanical and electrical relaxation for isotropic bodies,Proc. Vibr. Probl 4, 1-12.

[17] Paria, G., 1962, July. On magneto-thermo-elastic plane waves. In Mathematical Proceedings of the Cambridge Philosophical Society (Vol. 58, No. 3, pp. 527-531). Cambridge University Press.

[18] Purushothama, C.M., 1965, October. Magneto-thermo-elastic plane waves. In Mathematical Proceedings of the Cambridge Philosophical Society (Vol. 61, No. 4, pp. 939-944). Cambridge University Press.

[19] Singh, B. and Yadav, A.K., 2016. Plane Waves in a Rotating Monoclinic Magnetothermoelastic Medium. Journal of Engineering Physics and Thermophysics, 89(2), pp.428-440.

[20] Landersj, C., Hg, C., Maliniak, A. and Widmalm, G., 2000. NMR investigation of a tetrasaccharide using residual dipolar couplings in dilute liquid crystalline media: Effect of the environment. The Journal of Physical Chemistry B, 104(23), pp.5618-5624.

[21] Chattopadhyay, A., Gupta, S., Singh, A.K. and Sahu, S.A., 2009. Propagation of shear waves in an irregular magnetoelastic monoclinic layer sandwiched between two isotropic half-spaces. International Journal of Engineering, Science and Technology, 1(1), pp.228-244.

[22] Zhdanov, Viktor Mikhalovich. 2014 Transport processes in multicomponent plasma. CRC Press.

[23] Alfven H., 1942. On existence of electromagnetic-hydrodynamic waves: Ark Mat Astron Fys A29: pp 1-7.

[24] Khan A.A., Zaman A. and Yaseen S., 2018. Impact of two relaxation times on thermal, $\mathrm{P}$ and SV waves at interface with magnetic field and temperature dependent elastic moduli, Results in Physics 8 pp 324-335.

[25] Achenbach J.D., 1987. Wave propagation in elastic Solids, Vol (16) North Holland Series in Applied Mathematics and Mechanics.

[26] Lide, David R. 2005. Magnetic susceptibility of the elements and inorganic compounds. CRC handbook of chemistry and physics 86: 130-135. 